\title{
RPL13A as a reference gene for normalizing mRNA transcription of ovarian cancer cells with paclitaxel and 10-hydroxycamptothecin treatments
}

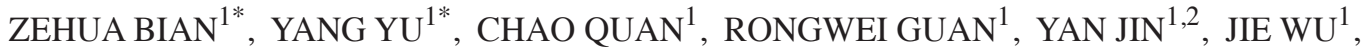 \\ LIDAN XU ${ }^{1}$, FENG CHEN $^{1}$, JING BAI $^{1}$, WENJING SUN ${ }^{1}$ and SONGBIN FU ${ }^{1,2}$ \\ ${ }^{1}$ Laboratory of Medical Genetics, Harbin Medical University, Harbin, Heilongjiang 150081; \\ ${ }^{2}$ Key Laboratory of Medical Genetics, Harbin Medical University, Heilongjiang Higher Education Institutions, \\ Harbin, Heilongjiang 150081, P.R. China
}

Received July 25, 2013; Accepted December 3, 2014

DOI: $10.3892 / \mathrm{mmr} .2014 .3108$

\begin{abstract}
Gene transcription analysis is important in cancer research, and reverse transcription-quantitative polymerase chain reaction (RT-qPCR) has been demonstrated to be an effective method to evaluate gene transcription in cancer. RT-qPCR requires an internal reference gene with a consistent level of mRNA transcription across various experimental conditions. However, it has been suggested that different treatments, including anticancer therapy, may influence the transcriptional stability of internal reference genes. Paclitaxel (PTX) and 10-hydroxycamptothecin (HCPT) are widely used to treat various types of cancer, and a suitable internal reference gene is required in order to analyze the transcription profiles of the cells following treatment. In the current study, the transcriptional stability of 30 candidate reference genes was investigated in cancer cells following treatment with PTX and HCPT. The two ovarian cancer cell lines, UACC-1598 and SKOV3, were treated with PTX and HCPT for 24 and $48 \mathrm{~h}$, and the transcriptional levels of the candidate reference genes were subsequently evaluated by RT-qPCR analysis. The transcriptional stability of the selected genes was then analyzed using qbase ${ }^{+}$and NormFinder software. A total of 9 genes were demonstrated to exhibit high transcriptional stability and one of these genes, ribosomal protein L13a (RPL13A), was identified to exhibit high transcriptional stability in every group. The current study identified various reference genes suitable under different circumstances, while RPL13A was indicated to be the most suitable reference gene
\end{abstract}

Correspondence to: Dr Songbin Fu, Laboratory of Medical Genetics, Harbin Medical University, 157 Baojian Road, Nangang, Harbin, Heilongjiang 150081, P.R. China

E-mail: fusb@ems.hrbmu.edu.cn; fusongbin@yahoo.com

Key words: internal reference gene, cancer, paclitaxel, reverse transcription-quantitative polymerase chain reaction, 10-hydroxycamptothecin for analyzing the transcription profile of ovarian cancer cells following treatment with PTX and HCPT.

\section{Introduction}

Gene transcription analysis is important in understanding the gene transcription profile in order to reveal complex mechanisms involved in disease initiation, progression and drug resistance $(1,2)$. Reverse transcription-quantitative polymerase chain reaction (RT-qPCR) is a sensitive method for the analysis of gene transcription in tissues or cells. This method requires reference genes that are not differentially transcribed across various tissues and experimental conditions, in order to evaluate target gene transcription (3). However, previous studies have suggested that several reference genes have variable levels of transcription, and that different treatments may influence the stability of the reference genes: Schmittgen and Zakrajsek (4) reported that the transcription of ACTB and GAPDH increased significantly following serum stimulation and that the most suitable internal control genes for this experimental condition were B2M and 18S RNA. Caradec et al (5) observed that certain cell lines required multiple different internal reference genes at different oxygen concentrations. This suggests that the utilization of certain internal reference control genes may adversely affect results and that suitable control reference genes should be determined to successfully analyze gene transcription in any experimental context. An additional study demonstrated that experimental results were highly dependent on the reference gene used (6).

In cancer research and clinical studies, paclitaxel (PTX) has been widely used to improve patient prognosis in the treatment of various types of gynecological cancer, including ovarian, cervical and endometrial cancer, in addition to breast, gastric and non-small-cell lung cancer (7-9). PTX interferes with spindle microtubules, resulting in depolymerization delay, which then triggers cell cycle arrest and cellular apoptosis (10). PTX is also able to directly bind to Bcl-2 and induce apoptosis (11). Camptothecin (CPT), a chemotherapeutic drug, has been reported to be efficient at inhibiting the growth of ovarian, hepatic, gastric, colorectal 
and breast cancer (12-16). CPT and its derivatives are able to inhibit the replication and transcription of DNA and mitosis by acting on type-I DNA topoisomerase $(17,18)$. The CPT derivative, 10-hydroxycamptothecin (HCPT) exhibits increased activity and is less toxic than other derivatives, which are all widely used and studied anticancer drugs. Therefore, if these anticancer treatments influence the transcriptional stability of the internal reference gene, the results of the investigation may be adversely affected. Thus, in the current study the transcriptional stabilities of several candidate reference genes were investigated, in order to determine the appropriate reference gene for the quantification of alterations in gene transcription following treatment of cancer cells with PTX and HCPT.

\section{Materials and methods}

Cell lines and cell culture. The human ovarian cancer cell line UACC-1598 was obtained from Dr Xin-Yuan Guan at the University of Hong Kong (Pok Fu Lam, Hong Kong). The human ovarian cancer cell line SKOV3 was obtained from the Cell Bank of the Type Culture Collection of the Chinese Academy of Sciences (Shanghai, China). These cells were cultured in RPMI-1640 (Invitrogen Life Technologies, Grand Island, NY, USA) containing $10 \%$ fetal bovine serum at $37^{\circ} \mathrm{C}$ in a $5 \% \mathrm{CO}_{2}$ humidified atmosphere. UACC-1598 and SKOV3 cells were treated with $0.5 \mathrm{ng} / \mu 1 \mathrm{PTX}\left(\mathrm{IC}_{50} \times 0.5\right.$; Knowshine Pharmachemicals, Inc., Shanghai, China) and $1.0 \mathrm{ng} / \mu \mathrm{l} \mathrm{HCPT}\left(\mathrm{IC}_{50} \times\right.$ 0.5; Knowshine Pharmachemicals, Inc.) for 24 and $48 \mathrm{~h}$ each.

Total RNA isolation and RT. Total RNA from the cultured cells was extracted using TRIzol reagent (Invitrogen Life Technologies) according to the manufacturer's instructions. The integrity of RNA was confirmed by electrophoresis (Bio-Rad Laboratories, Inc., Hercules, CA, USA) on a $1 \%$ agarose gel (Gene Tech Co., Ltd, Shanghai, China). The concentrations of the isolated RNA were determined by measuring the $\mathrm{A}_{260} / \mathrm{A}_{280}$ and $\mathrm{A}_{260} / \mathrm{A}_{230}$ absorbance ratios with a DU $800 \mathrm{spec}$ trophotometer (Beckman Coulter, Brea, CA, USA).

The concentration of RNA was adjusted to $500 \mathrm{ng} / \mu 1$ with nuclease-free water (Sigma-Aldrich, St. Louis, MO, USA). A total of $1 \mu \mathrm{g}$ total RNA was reverse-transcribed into cDNA using Transcriptor Fisrt Strand cDNA Synthesis Kit (Roche Diagnostics, Indianapolis, IN, USA) in a total reaction volume of $20 \mu \mathrm{l}$ by incubation at $50^{\circ} \mathrm{C}$ for $60 \mathrm{~min}$, followed by $85^{\circ} \mathrm{C}$ for $5 \mathrm{~min}$.

Gene selection, primer design and efficiency evaluation. A total of 30 candidate cancer-associated internal control genes were selected according to previous studies (Table I) (19-25) Certain primer sequences were selected from these studies and others were designed using Gene Runner software, version 3.05 (www.generunner.net); they are summarized in Table I. The RT-qPCR efficiencies for all primers were detected using serial 10-fold dilutions of the same cDNA sample. Amplification efficiencies were calculated automatically from raw fluorescence data by the LightCycler 480 software (version 1.5 ) in the Light Cycler $^{\circledR} 480$ Real-Time PCR System (Roche Diagnostics).
$R T-q P C R$. RT-qPCR was performed using the LightCycler 480 SYBR Green I Master mix (Roche Diagnostics). The RT-qPCR reaction mix consisted of $0.5 \mu \mathrm{M}$ forward and reverse primers (Table I), $10 \mu$ l Master mix, 100 ng cDNA and nuclease-free water up to a $20 \mu \mathrm{l}$ reaction volume. All experiments were performed in triplicate and a no-template control (reaction mix without cDNA) was used in each assay. RT-qPCR assays were conducted at $95^{\circ} \mathrm{C}$ for a 5 min pre-incubation, then 45 cycles of denaturation at $95^{\circ} \mathrm{C}$ for $10 \mathrm{sec}$, primer reannealing at $58-62^{\circ} \mathrm{C}$ for $20 \mathrm{sec}$ and extension at $72^{\circ} \mathrm{C}$ for $30 \mathrm{sec}$. The RT-qPCR reaction was followed by melting curve analysis at $95^{\circ} \mathrm{C}$ for $5 \mathrm{sec}$ and $65^{\circ} \mathrm{C}$ for $1 \mathrm{~min}$.

Data analysis. For a stability comparison of the candidate reference genes, two pieces of software were used, qbase $^{+}$(version 2.0; http://www.biogazelle.com/qbaseplus; Biogazelle, Ghent, Belgium) and the Microsoft Excel add-in program NormFinder (version 19; http://moma.dk/normfinder-software; Department of Molecular Medicine, Aarhus, Denmark).

\section{Results}

RNA extraction and gene transcription. Total RNA was extracted from the two cell lines following treatment with PTX and HCPT for 0, 24 and $48 \mathrm{~h}$, then the integrity was confirmed by the ratios of $28 \mathrm{~S}$ to $18 \mathrm{~S}$ ribosomal RNA with agarose gel electrophoresis (data not shown). It was determined that all samples had $\mathrm{A}_{260} / \mathrm{A}_{280}$ ratios $>1.9$ and $\mathrm{A}_{260} / \mathrm{A}_{230}$ ratios $>1.5$, which indicates that the extracted RNA was of sufficient purity for the experiments. The levels of the 30 candidate genes were then evaluated using RT-qPCR in the samples of ovarian cancer cells treated with PTX or HCPT. The cycle threshold values for these genes were 12.11-28.65, which indicated that every selected gene had appropriate transcription (data not shown). Preliminary analysis of the transcriptional stability of each gene with or without treatment was conducted using a one-way analysis of variance. A total of 12 genes were identified in which the levels of transcription remained stable following treatment with PTX, and 10 transcriptionally stable genes were identified following HCPT treatment. The selected genes, which are presented in Table I, were further analyzed using the gene transcription software described.

Analysis of the stability of gene transcription in $U A C C-1598$ cells. qbase ${ }^{+}$software was used to identify suitable reference genes via the analysis of the transcriptional stability of the selected genes in ovarian cancer cells treated with PTX and HCPT. qbase $^{+}$software utilizes a pairwise comparison of variation to compute a geNorm $\mathrm{M}$ value for each gene and an average geNorm M. A geNorm $M$ value $<$ the average geNorm $M$ value was considered to indicate high transcriptional stability. The lower the geNorm $\mathrm{M}$ value of the internal reference gene is, the greater the transcriptional stability it will have. qbase ${ }^{+}$software also calculates a geNorm $\mathrm{V}$ value, which represents the optimal number of reference genes to combine in one experiment. In the UACC-1598 cells treated with PTX, qbase $^{+}$analysis calculated the geNorm $\mathrm{M}$ for each gene, with an average of 0.200 (Fig. 1A). The genes with a lower than average geNorm $M$ value [B2M ( $\beta$-2-microglobulin), ribosomal protein L13a (RPL13A), GAPDH and phosphoglycerate 


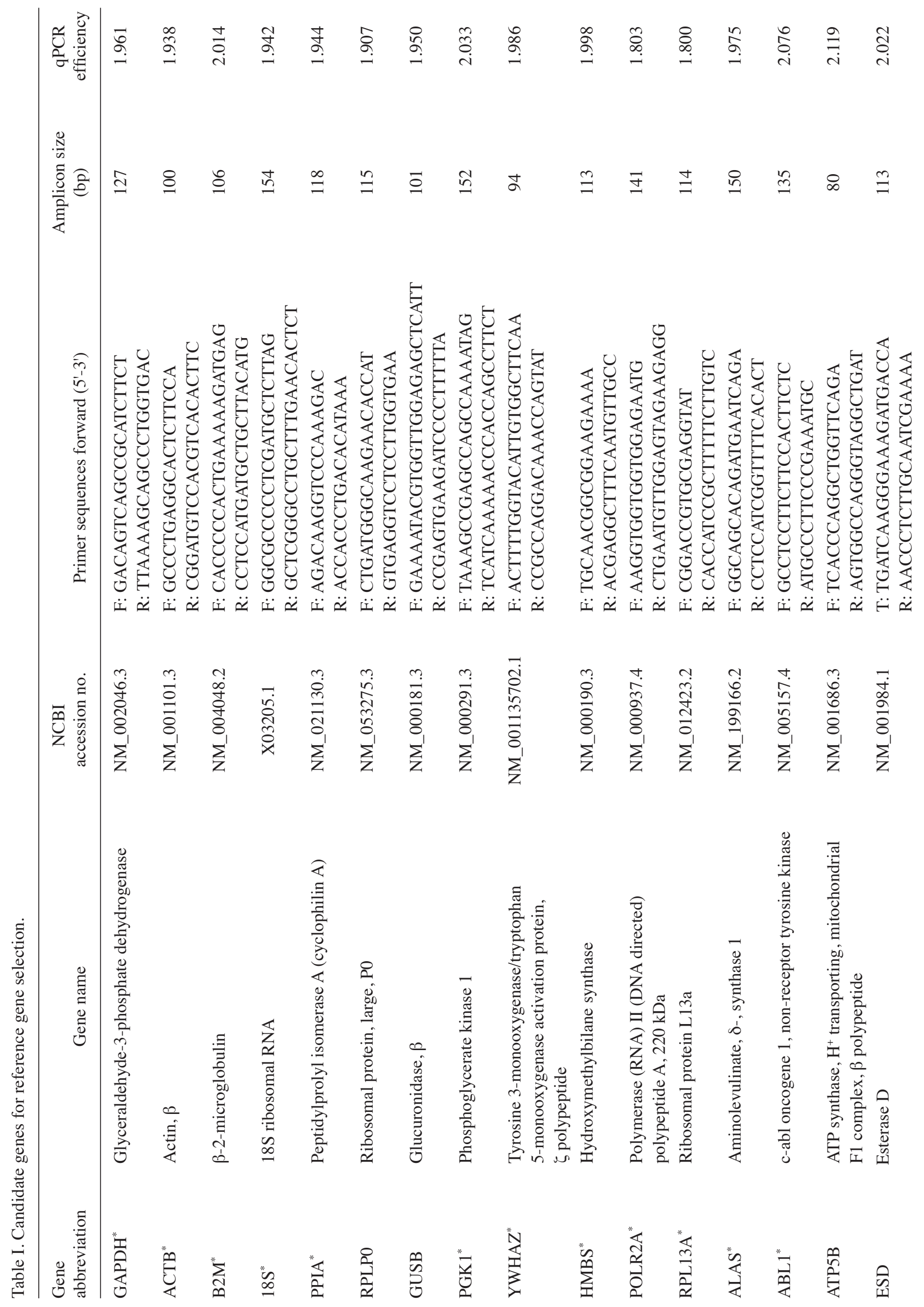




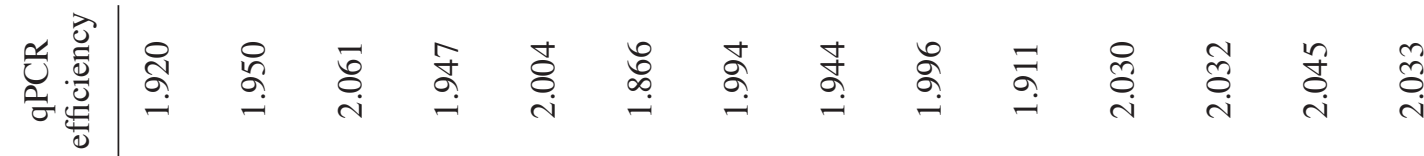
$\stackrel{\mathbb{N}}{\mathrm{m}}$ 安

$\underset{⿱}{\circlearrowright}$

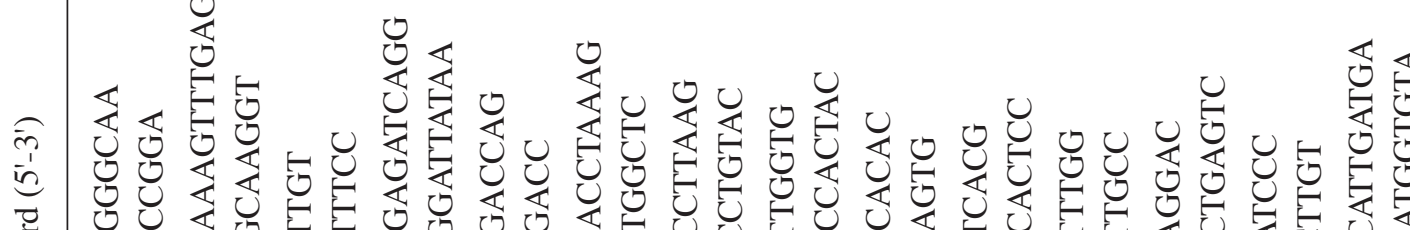

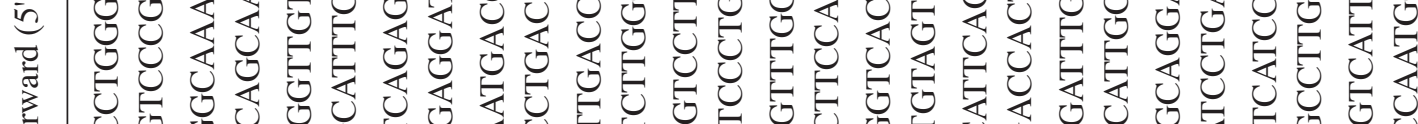

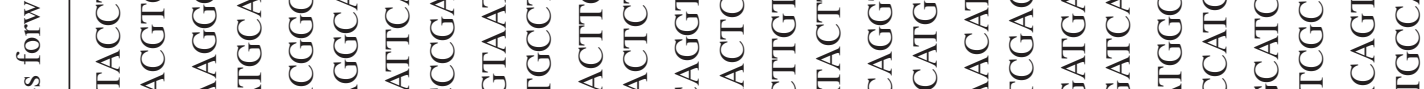

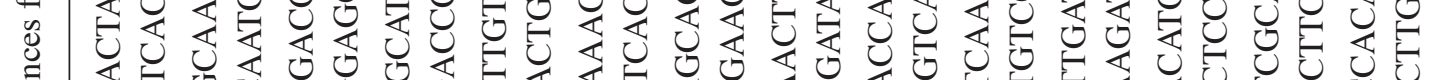

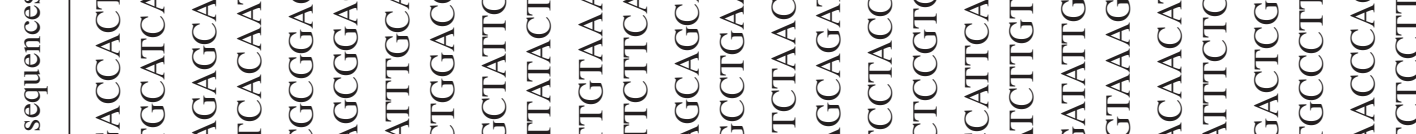

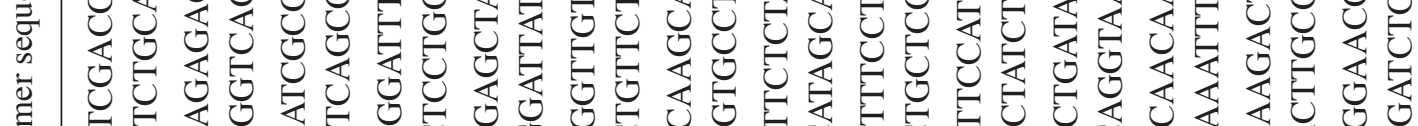

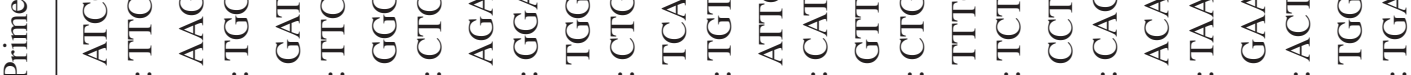

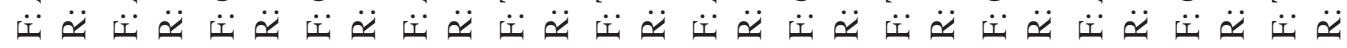

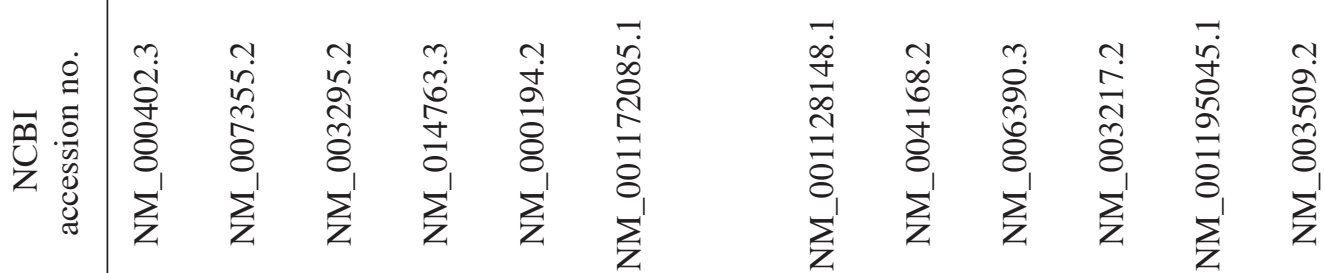

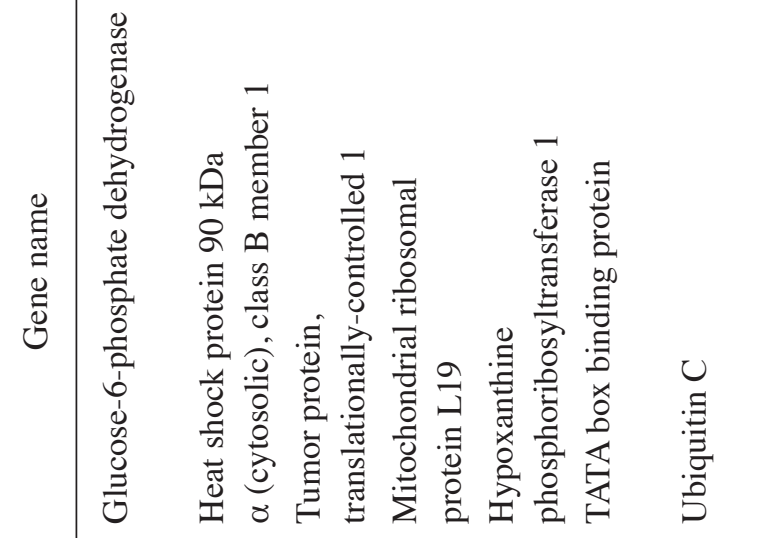

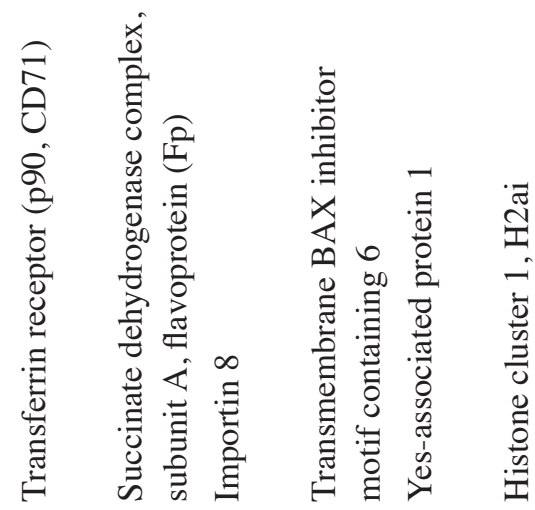

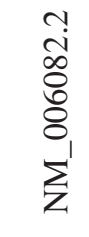

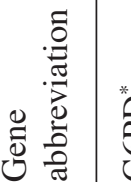

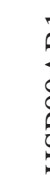

究 
kinase 1 (PGK1)] were considered to have high transcriptional stability. Furthermore, B2M, which had the lowest geNorm M value (0.109) was identified as the most stable reference gene in UACC-1598 cells treated with PTX. The data from UACC-1598 cells was also analyzed using NormFinder software which yielded similar results, identifying B2M as the optimal internal reference gene (data not shown). The optimal number of genes selected in transcriptional studies was identified to be two, the results indicating that it is not necessary to include more than two control genes for analysis (Fig. 1B). The optimal normalization factor was calculated as the geometric mean of the reference combination B2M and RPL13A. Determination of the optimal number of control genes for normalization was performed on the basis of a pair-wise variation $(\mathrm{Vn} / \mathrm{n}+1)$ analysis. $\mathrm{V} 2 / 3$ was the first value typically lower than the cutoff value 0.150 , indicating that there was no need to include the third gene in order to determine the optimal number of reference genes.

For UACC-1598 cells treated with HCPT, qbase ${ }^{+}$analysis demonstrated that five genes with a geNorm $M$ lower than average (GAPDH, RPL13A, G6PD, ALAS and ABL1) had high reference target stability (Fig. 2B). GAPDH exhibited the lowest geNorm M value (0.090) and thus was identified as the most stable reference gene in HCPT-treated UACC-1598 cells (Fig. 2A). The optimal normalization factor was two, as illustrated in Fig. 2B and the best reference gene combination was GAPDH and RPL13A (Fig. 2B).

The reference genes with high transcriptional stability in PTX- or HCPT-treated UACC-1598 cells varied, suggesting that stimulation with different chemotherapeutic drugs has a varied effect on the gene transcription profile in the same cell line. The widely used reference gene ACTB presented poor transcriptional stability in PTX- and in HCPT-treated cells. However, in PTX- and HCPT-treated UACC-1598 cells, RPL13A remained transcriptionally stable, thus it was concluded that RPL13A is a suitable reference gene in UACC-1598 cells for gene transcription analysis.

Analysis of the stability of gene transcription in SKOV3 cells. The transcriptional stabilities of the selected genes were then investigated in the SKOV3 ovarian cancer cell line following treatment with PTX and HCPT. qbase ${ }^{+}$analysis revealed that the average geNorm M was 0.500 (Fig. 3A). The RPL13A, IPO8, YWHA and ALAS genes all presented geNorm M values lower than the average, and exhibited high transcriptional stability following treatment with PTX. The optimal reference gene combination was observed to be RPL13A and IPO8 (Fig. 3B).

In the analysis of SKOV3 cells treated with HCPT, qbase ${ }^{+}$ analysis identified four genes that presented high transcriptional stability (RPL13A, G6PD, PPIA and B2M; Fig. 4A), and the best reference gene combination was RPL13A and G6PD (Fig. 4B).

The results obtained in SKOV3 cells were consistent with those of UACC-1598 cells, with RPL13A exhibiting a high transcriptional stability in the two cell types, when treated with PTX or HCPT. Together, these results suggest that different cell lines originating from the same organ may exhibit different transcription profiles following drug treatment. Although the different cell lines may exhibit stable transcription of different internal reference genes, RPL13A
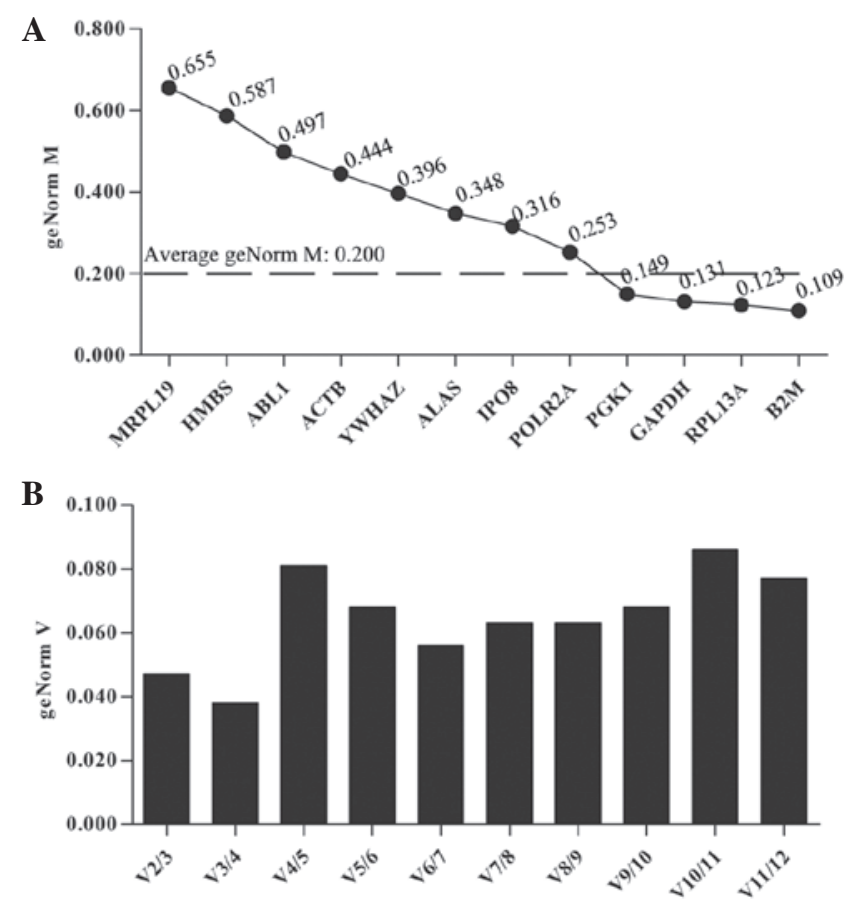

Figure 1. Ranking of the internal reference genes in UACC-1598 cells treated with PTX. (A) Transcriptional stability of the selected genes was evaluated by qbase ${ }^{+}$software. A total of four genes were identified to have high transcriptional stability (average geNorm $\mathrm{M}<$ the average). $\mathrm{X}$ axes, internal reference genes. (B) qbase ${ }^{+}$analysis computes a geNorm $\mathrm{V}$ as the variability between sequential normalization factors to determine the optimal number of reference genes. Additional genes are included when the variation exceeds the cutoff value, which is typically set at 0.150 . V2/3 was $<0.150$ thus the optimal normalization factor is 2 , and an appropriate combination of reference targets is RPL13A and B2M. X axes, pairwise variations.
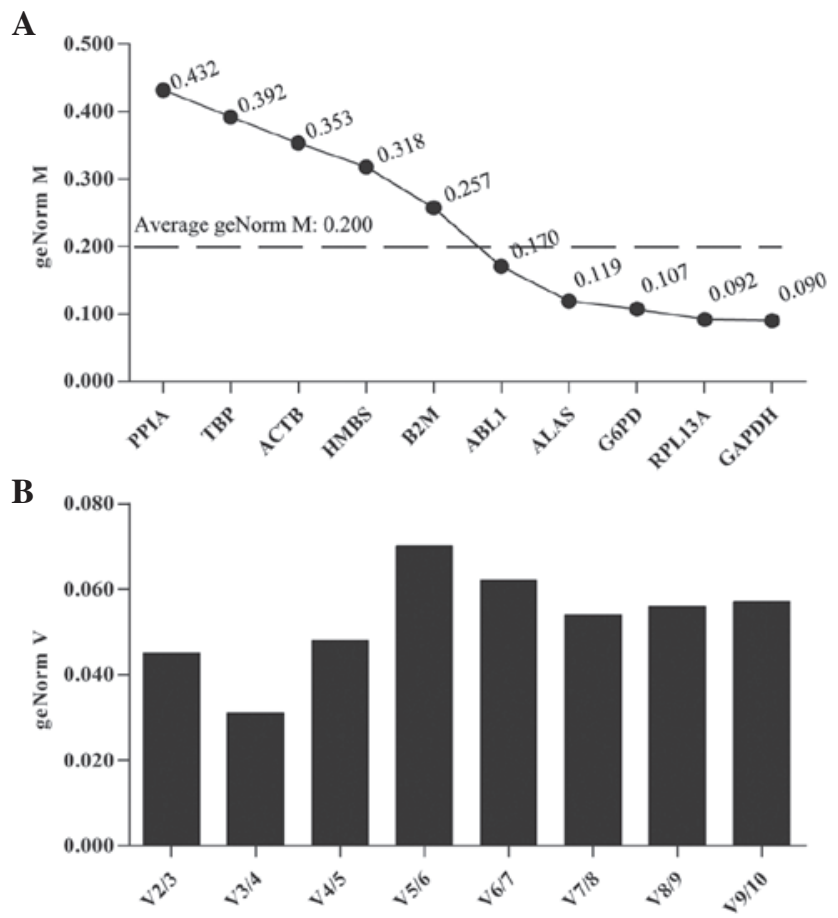

Figure 2. Ranking of the internal reference genes in UACC-1598 cells treated with HCPT. (A) Transcriptional stability of the selected genes was evaluated using qbase ${ }^{+}$software. A total of five genes were identified to have high target reference stability (average geNorm $\mathrm{M}<$ the average). $\mathrm{X}$ axes, internal reference genes. (B) The optimal normalization factor can be calculated as the geometric mean of reference targets GAPDH and RPL13A. X axes, pairwise variations. 


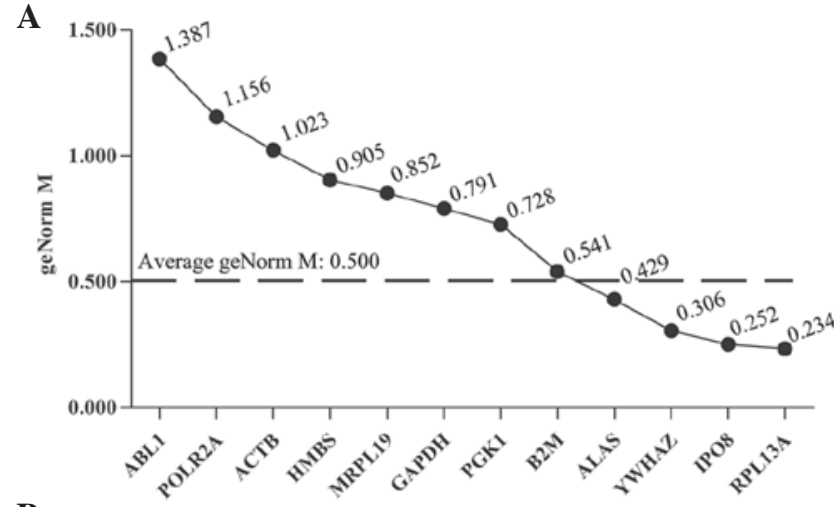

B

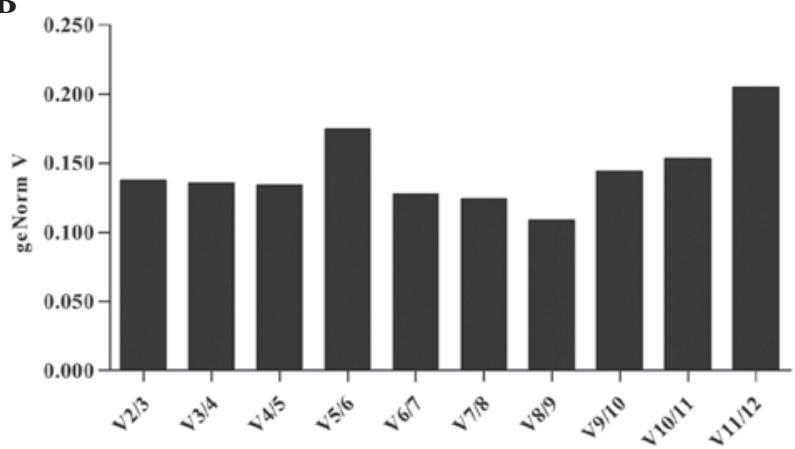

Figure 3. Ranking of the internal reference genes based on qbase ${ }^{+}$software in the samples of SKOV3 cells treated with PTX. (A) A total of four genes were identified to have high target reference stability (average geNorm $\mathrm{M}<$ the average). (B) Evaluation of the optimum combination of reference genes for normalization. $\mathrm{V} 2 / 3<0.150$ indicates the optimal normalization factor is 2 and that an appropriate combination of reference targets is IPO8 and RPL13A.

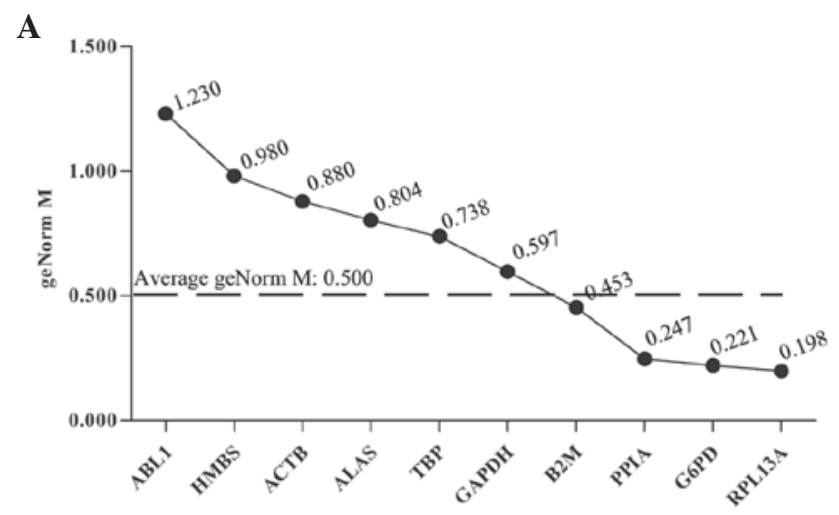

B

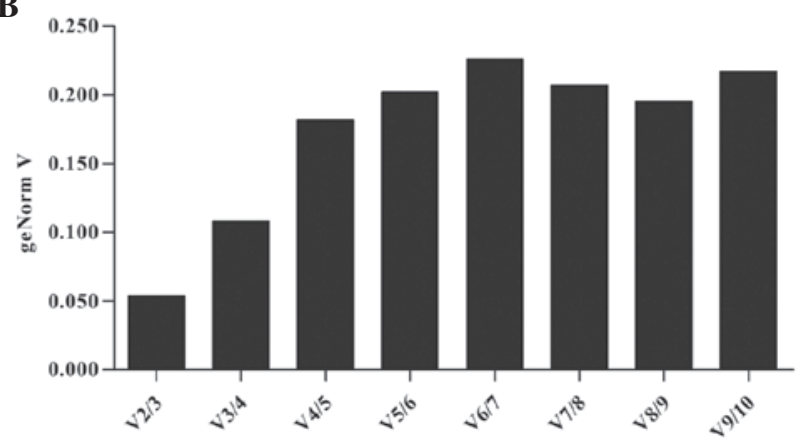

Figure 4. Ranking of the internal reference genes based on qbase ${ }^{+}$software in the samples of SKOV3 cells treated with HCPT. (A) A total of four genes were identified to have high target reference stability (average geNorm $\mathrm{M}<$ the average). (B) Evaluation of the optimum of reference genes for normalization. V2/3 $<0.150$ indicates the optimal normalization factor is 2 and that an appropriate combination of reference targets is G6PD and RPL13A.
Table II. Stability of internal reference genes in UACC-1598 and SKOV3 cells under different conditions.

\begin{tabular}{lccccc}
\hline & \multicolumn{2}{c}{ UACC-1598 } & & \multicolumn{2}{c}{ SKOV3 } \\
\cline { 2 - 3 } \cline { 5 - 6 } Gene & PTX & HCPT & & PTX & HCPT \\
\hline B2M & + & & & + \\
RPL13A & + & + & & + & + \\
GAPDH & + & + & & & \\
PGK1 & + & & & & + \\
G6PD & & + & & + \\
ALAS & & & & + & \\
IPO8 & & & & + & + \\
YWHAZ & & & & + & \\
PPIA & & & & & +
\end{tabular}

+ indicates the genes have transcriptional stability in that condition. PTX, paclitaxel; HCPT, 10-hydroxycamptothecin.

is a suitable reference gene for gene transcription analysis in ovarian cancer cells following PTX or HCPT treatments.

\section{Discussion}

RT-qPCR is widely used to analyze gene transcription in cells. To evaluate the differences in the transcription of target genes, it is necessary to eliminate the differences in RNA quality and quantity, in addition to variations in RT efficiency. One method to overcome this problem is to adjust the transcription of the target genes using an internal reference gene. An ideal reference gene has consistent transcription in all experimental conditions. A previous study that focused on internal reference genes for ovarian cancer identified GUSB, PPIA and TBP as stably transcribed genes and suggested that GUSB and PPIA be used in combination as internal reference genes to normalize the transcriptional levels of other target genes (26). An additional study suggested that the genes RPL4, RPLP0 and HSPCB presented the most stable transcription in ovarian cancer tissues, and thus recommended RPL4 and RPLP0 as reference genes for normalization (25). This research illustrates that there are different appropriate reference genes for different ovarian cancer samples. These observations support the theory that following anticancer drug treatment, ovarian cancer cells require different reference genes.

In the current study, the transcriptional levels of 30 candidate genes were analyzed, and the genes that transcribed differently following drug treatments were eliminated from the search at a preliminary stage. The stability of transcription for the selected genes was then evaluated using qbase ${ }^{+}$ and NormFinder software. Approximately four genes were identified to have high transcriptional stability in each experimental group. The identified genes in the different groups were compared and a total of nine genes were indicated to have high transcriptional stability. In addition, there were five genes that presented high transcriptional stability in $>2$ groups. RPL13A was observed to exhibit high transcriptional stability in all groups (Table II). It was concluded from 
the data collected that treatment with PTX and HCPT did not influence the transcriptional stability of RPL13A. The results of the present study suggest that the transcriptional stability of genes fluctuates when the cells are exposed to different conditions, such as drug treatment. A previous study evaluated the transcriptional stability of 11 genes following treatment with tamoxifen, revealing that gene transcription was significantly altered except for that of RPL13A, TFRC and GUSB (27). This observation suggests that treatment with anticancer drugs may influence the transcriptional stability of the majority of internal reference genes, which is consistent with the observations of the current study. With pharmacological stimulation, the transcriptional profile of cancer cells may be altered greatly. The present study demonstrated that with the exception of the gene RPL13A, which had comparable transcriptional stability in the two cell lines investigated, the other genes were ranked differently in their transcriptional stability prior to and subsequent to drug treatment. The results support the theory that genes are not stably transcribed in all types of carcinoma, including cells from similar tissues, following treatment with the same drug. In view of the fact that different drugs influence the transcriptional stability of genes in different ways, it is necessary to determine the best reference gene prior to analysis of the transcription profile of cells receiving treatment. The current study demonstrated for the first time that following treatment of ovarian cancer cells with PTX and HCPT, different reference genes are stably transcribed in the different cells. It is notable that RPL13A is a suitable reference gene for normalizing gene expression of ovarian cancer cells following anticancer drug treatment.

\section{Acknowledgements}

The present study was supported by the International Science \& Technology Cooperation Program of China (grant no. 2013DFA31610); the Program for Changjiang Scholars and Innovative Research Team in University (grant no. IRT1230); the New Century Support Program for the Excellent Scholar, Ministry of Education of China (grant nos. NCET-10-0149 and NCET-11-0954); and the National Natural Science Foundation of China (grant no. 81272288).

\section{References}

1. Pal S, Gupta R and Davuluri RV: Alternative transcription and alternative splicing in cancer. Pharmacol Ther 136: 283-294, 2012.

2. Richardson A and Kaye SB: Drug resistance in ovarian cancer: the emerging importance of gene transcription and spatio-temporal regulation resistance. Drug Resist Updat 8: 311-321, 2005.

3. Lin Y, Zhang C, Lan H, Gao S, et al: Validation of potential reference genes for qPCR in maize across abiotic stresses, hormone treatments, and tissue types. PLoS One 9: e95445, 2014.

4. Schmittgen TD and Zakrajsek BA: Effect of experimental treatment on housekeeping gene expression: validation by real-time, quantitative RT-PCR. J Biochem Biophys Methods 46: 69-81, 2000.

5. Caradec J, Sirab N, Keumeugni C, et al: 'Desperate house genes': the dramatic example of hypoxia. Br J Cancer 102 1037-1043, 2010.
6. Dheda K, Huggett JF, Chang JS, et al: The implications of using an inappropriate reference gene for real-time reverse transcription PCR data normalization. Anal Biochem 344: 141-143, 2005.

7. Montana M, Ducros C, Verhaeghe P, Terme T, Vanelle P and Rathelot P: Albumin-bound paclitaxel: the benefit of this new formulation in the treatment of various cancers. J Chemother 23: 59-66, 2011.

8. Wang N, Zhang H, Yao Q, Wang Y, Dai S and Yang X: TGFBI promoter hypermethylation correlating with paclitaxel chemoresistance in ovarian cancer. J Exp Clin Cancer Res 31: 6, 2012.

9. Socinski MA, Okamoto I, Hon JK, et al: Safety and efficacy analysis by histology of weekly nab-paclitaxel in combination with carboplatin as first-line therapy in patients with advanced non-small-cell lung cancer. Ann Oncol 24: 2390-2396, 2013.

10. Amos LA and Löwe J: How Taxol stabilises microtubule structure. Chem Biol 6: R65-R69, 1999.

11. Ferlini C, Cicchillitti L, Raspaglio G, et al: Paclitaxel directly binds to Bcl-2 and functionally mimics activity of Nur77. Cancer Res 69: 6906-6914, 2009.

12. Ping YH, Lee HC, Lee JY, et al: Anticancer effects of low-dose 10-hydroxycamptothecin in human colon cancer. Oncol Rep 15: 1273-1279, 2006.

13. Zhang XW, Qing $\mathrm{C}$ and $\mathrm{Xu} \mathrm{B}$ : Apoptosis induction and cell cycle perturbation in human hepatoma hep $\mathrm{G} 2$ cells by 10-hydroxycamptothecin. Anticancer Drugs 10: 569-576, 1999.

14. Lesueur-Ginot L,Demarquay D, Kiss R, et al: Homocamptothecin, an E-ring modified camptothecin with enhanced lactone stability, retains topoisomerase I-targeted activity and antitumor properties. Cancer Res 59: 2939-2943, 1999.

15. Shoji T, Takatori E, Kaido Y, et al: A phase I study of irinotecan and pegylated liposomal doxorubicin in recurrent ovarian cancer (Tohoku Gynecologic Cancer Unit 104 study). Cancer Chemother Pharmacol 73: 895-901, 2014.

16. Rino Y, Yukawa N, Sato T, et al: Phase II study on the combination of irinotecan plus cisplatin as a second-line therapy in patients with advanced or recurrent gastric cancer. Mol Clin Oncol 1: 749-752, 2013.

17. Hertzberg RP, Caranfa MJ and Hecht SM: On the mechanism of topoisomerase I inhibition by camptothecin: evidence for binding to an enzyme-DNA complex. Biochem 28: 4629-4638, 1989.

18. Fukada M: Action of camptothecin and its derivatives on deoxyribonucleic acid. Biochem Pharmacol 34: 1225-1230, 1985.

19. Cicinnati VR, Shen Q, Sotiropoulos GC, Radtke A, Gerken G and Beckebaum S: Validation of putative reference genes for gene expression studies in human hepatocellular carcinoma using real-time quantitative RT-PCR. BMC Cancer 8: 350, 2008.

20. Gresner P, Gromadzinska J and Wasowicz W: Reference genes for gene expression studies on non-small cell lung cancer. Acta Biochim Pol 56: 307-316, 2009.

21. Valceckiene V, Kontenyte R, Jakubauskas A and Griskevicius L: Selection of reference genes for quantitative polymerase chain reaction studies in purified $\mathrm{B}$ cells from $\mathrm{B}$ cell chronic lymphocytic leukaemia patients. Br J Haematol 151: 232-238, 2010.

22. Ferreira E and Cronjé MJ: Selection of suitable reference genes for quantitative real-time PCR in apoptosis-induced MCF-7 breast cancer cells. Mol Biotechnol 50: 121-128, 2012.

23. Andersen CL, Jensen JL and Ørntoft TF: Normalization of real-time quantitative reverse transcription-PCR data: a model-based variance estimation approach to identify genes suited for normalization, applied to bladder and colon cancer data sets. Cancer Res 64: 5245-5250, 2004.

24. Ohl F, Jung M, Xu C, et al: Gene expression studies in prostate cancer tissue: which reference gene should be selected for normalization? J Mol Med (Berl) 83: 1014-1024, 2005.

25. Fu J, Bian L, Zhao L, et al: Identification of genes for normalization of quantitative real-time PCR data in ovarian tissues. Acta Biochim Biophys Sin (Shanghai) 42: 568-574, 2010.

26. Li YL, Ye F, Hu Y, Lu WG and Xie X: Identification of suitable reference genes for gene expression studies of human serous ovarian cancer by real-time polymerase chain reaction. Anal Biochem 394: 110-116, 2009.

27. Shah KN and Faridi JS: Estrogen, tamoxifen, and Akt modulate expression of putative housekeeping genes in breast cancer cells. J Steroid Biochem Mol Biol 125: 219-225, 2011. 